\title{
Bericht vom 11. Walter-Brendel-Kolleg für J. Litmathe Transplantationsmedizin vom 7. - 12. März 2004
}

\author{
Report on the 11th Walter-Brendel-Lecture for Transplantation, \\ March 7th-12th, 2004
}

Vom 7.-12. März 2004 fand in Wildbad Kreuth am Tegernsee einmal mehr das so genannte Walter-Brendel-Kolleg statt, benannt nach dem Pionier der Organtransplantation und Gründungsvater des Instituts für chirurgische Forschung an der Ludwig-Maximilians-Universität München. Das Kolleg versteht sich als Forum zum Unterricht in den Grundlagen der Organtransplantation und richtet sich vornehmlich an junge Nachwuchsärzte, die sich diesem Spezialgebiet der Medizin besonders widmen. Namhafte Referenten waren auch diesmal geladen, um dem etwa 50 Köpfe zählenden Auditorium aus dem gesamten Bundesgebiet sowie aus Österreich und der Schweiz die neuesten Entwicklungen der Transplantationsmedizin zu vermitteln und ihnen in z.T. lebhaften Diskussionen Rede und Antwort zu stehen. Die qualitative Hochwertigkeit dieses Kollegs wird einerseits durch die langjährige klinische Erfahrung der Referenten, andererseits durch die selektive Auswahl besonders motivierter Hörer aus den verschiedensten konservativen und operativen Zentren sichergestellt.

Nachdem als Eingangslesung eine interessante Rückschau auf das Institut für chirurgische Forschung und Walter Brendel selbst gehalten worden war, wurde sich in der ersten zentralen Sitzung den grundlegenden Fragen der Ethik der Organtransplantation sowie den juristischen Aspekten gewidmet. Hierbei wurde insbesondere das Prinzip der Lebenswertindifferenz als ethische Basis betont, jedoch trägt der ausgeprägte Spendermangel in Deutschland entscheidend zur Beantwortung der Fragen der Organallokation bei, und zwar im Sinne einer höchstmöglichen Effizienz, wie nicht zuletzt vom Leiter der zentralen Vermittlungsstelle Eurotransplantat/Leiden herausgestellt wurde. Selbst die mehr oder minder klare gesetzliche Grundlage, die durch das Transplantationsgesetz in Deutschland geschaffen wurde, konnte nicht entscheidend zur Verbesserung des Spen- dermangels beitragen. Vor diesem Hintergrund gewinnt die Lebendorganspende zunehmend an Bedeutung. So beträgt der Anteil realisierbarer Organspenden pro eine Million Einwohner in Deutschland derzeit 23,4. Hier nimmt die Deutsche Stiftung Organtransplantation (DSO) einen zentralen Platz ein.

Neben den Grundlagen sowie auch den neuesten Entwicklungen im Bereich der Alloimmunität (z.B. Rolle des plazentaren IDOGens in Zusammenhang mit der Wirkung von Mitomycin) wurde in weiteren Sitzungen auch interessante Ansatzpunkte der Toleranzinduktion behandelt: Hier kommt löslichen HLA-Antigenen, insbesondere dem HLA-G Molekül eine entscheidende Funktion zu. Aber auch die Entwicklung synthetischer Zytokine, die die Signaltransduktion zu blockieren vermögen, ist Gegenstand der aktuellen Forschung.

In den folgenden Sitzungen traten Aspekte der Organischämie, -anoxie, des Reperfusionsschadens und die verschiedenen Formen von Abstoßungsreaktionen in den Mittelpunkt des Interesses: Insbesondere für die Lungenkonservierung ist der niedrige Kalium-Gehalt, was durch den Einsatz von EC-solution, die in 77\% der Zentren Verwendung findet, Berücksichtigung erfährt. Auch der protektive Effekt des wasserlöslichen Vitamin-E-Analogons Trolox bekommt in der Minderung des ischämischen bzw. Reperfusionsschadens vor allem der Lunge zunehmend Bedeutung.

Die weiteren Lesungen befassten sich mit der klinischen und experimentellen Immunsuppression. Die bekannte Basis-Chemotherapie bestehend aus Kortikosteroiden, Mycophenolat und/ oder Calcineurininhibitoren wurde ebenso beleuchtet wie auch neueste Entwicklungen bis hin zum FTY 720, das durch die Verminderung der peripheren Lymphozytenzahl einen sog. Ho- 
ming-Effekt herbeiführt. Als unerwünschte Arzneimittelwirkungen sind neben den opportunistischen Infekten bösartige Neubildungen (Lippen- und Hautsarkome sowie maligne Erkrankungen des lymphatischen Systems, sog. PTLD = post transplant lymphoproliferative disorder) allgemein bekannt.

Selbstverständlich sind auch die spezifischen klinischen Punkte der Transplantation einzelner oder mehrerer Organe berücksichtigt worden: Hier darf besonders die Lungen- bzw. kombinierte Herz-Lungentransplantation herausgestellt werden: Ein historischer Rückblick zeigt die erste Lungentransplantation im Jahre 1963 auf dem Boden eines Bronchial-Karzinoms. Nach einer anfänglich etwas zögernden Entwicklung hat die Anzahl fast kontinuierlich zugenommen; im Jahre 2003 sind in Deutschland erstmals über 200 Lungentransplantationen durchgeführt worden. Technische Details sind seit den sechziger Jahren wesentlich verbessert worden, wie z.B. die heutzutage oftmals nur noch notwendige Mini-Thorakotomie gegenüber dem früher praktizierten clam-shell-Zugang, der aktuell noch bei Re-Transplantationen mit starken Verwachsungen Verwendung findet, zeigt. Als wesentlicher prognostischer Faktor für das post-operative survival wurde der Aspekt der präoperativen Beatmung als negativer Prediktor betont: Hier zeigt sich eine Absterbe-Rate von etwa $50 \%$ nach $12-28$ Monaten. Noch schlechter stellt sich die Überlebensrate bei vorausgegangener Bronchusnahtinsuffizienz im Rahmen von Retransplantationen dar: Hier überleben weniger als 50\% der Patienten die ersten drei Jahre. Erwähnung fand zudem auch die Möglichkeit der ex-situ Lobektomie, die es heutzutage ermöglicht, akzeptable Transplantatvolumina bei Größenmismatches oder bei traumatisch beschädigten Abschnitten, z.B. einer isolierten Kontusion des Unterlappens nach vorausgegangenem Verkehrsunfall des Spenders in der Lage ist, qualitativ akzeptable grafts zu produzieren. Hinsichtlich der Immunsuppression zeigt sich eine Überlegenheit hinsichtlich des graft-survivals bei Mycophenolat gegenüber Azathioprin. Seit dem 15. März 2004 ist auch Certican ${ }^{\mathrm{TM}}$, das in seiner Grundstruktur starke Ähnlichkeit mit Sirolimus aufweist, für die Initialtherapie nach Lungentransplantation zugelassen. Ein interessanter Ver- gleich von double- und single-lung-Transplantationen zeigt während eines follow-up von 5 Jahren keine nennenswerten Unterschiede, jedoch danach signifikante Überlebensvorteile von double-lung-Transplantaten. Hinsichtlich des Auftretens maligner Tumoren nach Lungentransplantation zeigt eine Analyse der UNOS-Datenbank aus dem Zeitraum 1984 - 1999 in einem 1-Jahres-follow-up eine Inzidenz von 4,3\% und in einem 5-Jahres-follow-up eine Inzidenz von 7,7\%. Nach $1 \mathrm{Jahr}$ dominieren hierunter Lymphome mit 50,7\%, nach 5 Jahren jedoch Hauttumoren mit ebenfalls 50,7\%. Weitere Begleiterkrankungen (UNOS-Daten 1994 - 1999) und Komplikationen werden wie folgt zusammengefasst (Tab. 1):

Tab. 1 Begleiterkrankungen und Komplikationen nach Lungentransplantation; zusammengestellt von M. Strüber, Hannover

1 Jahr nach Lungen-Tx 5 Jahre nach Lungen-Tx

\begin{tabular}{lrr}
\hline arterielle Hypertonie & $48,6 \%$ & $61,1 \%$ \\
\hline Nierenfunktionsstörung & $11,6 \%$ & $14,7 \%$ \\
\hline chronische Dialyse & $1,2 \%$ & $3,8 \%$ \\
\hline Hyperlipidämie & $11,7 \%$ & $17,9 \%$ \\
\hline Diabetes mellitus & $15,4 \%$ & $14,3 \%$ \\
\hline Bronchiolitis obliterans & $11,6 \%$ & $32,4 \%$ \\
\hline
\end{tabular}

Insgesamt, auch unter Berücksichtigung der Transplantation extrathorakaler Organe, stellt das Walter-Brendel-Kolleg ein ausgewogenes Konzept zur Transplantationsschulung junger Mediziner dar, dessen regelmäßige Neuauflage von außerordentlichem Nutzen sowohl für den klinischen als auch wissenschaftlichen Nachwuchs ist. Den Organisatoren, Herrn Prof. Dr. Hopt, Freiburg und Herrn Prof. Dr. Abendroth, Ulm sei auf diesem Wege nochmals hohe Anerkennung ausgedrückt in der Hoffnung, dass dieses Seminar auch in Zukunft noch vielen in Weiterbildung befindlichen Ärzten offen stehen wird. 